\title{
A NOVEL ITERATIVE APPROACH FOR COMPLEX SINGLE-TONE FREQUENCY ESTIMATION
}

\author{
K.W. Chan and H. C. So \\ Department of Electronic Engineering, City University of Hong Kong, Kowloon, Hong Kong \\ email: k.w.chan@student.cityu.edu.hk,hcso@ee.cityu.edu.hk
}

\begin{abstract}
Based on linear prediction and weighted least squares, an iterative procedure for frequency estimation of a complex sinusoid in white noise is devised. The proposed approach, which utilizes the first-order as well as higher-order linear prediction terms simultaneously but does not require phase unwrapping, can be considered as a generalized version of the weighted linear predictor frequency estimator. Computer simulations are included to contrast the performance of the proposed algorithms with Cramér-Rao lower bound in one-dimensional and two-dimensional frequency estimation.
\end{abstract}

\section{INTRODUCTION}

Estimating the frequencies of a sinusoidal signal in noise has applications in many areas such as radar, sonar and communications. In this paper, we consider the most basic form of the frequency estimation problem, namely, finding the frequency of a pure complex tone in white noise. The one-dimensional (1-D) single tone model is

$$
x_{n}=s_{n}+q_{n}, \quad s_{n}=A e^{j(\omega n+\phi)}, \quad n=1,2, \ldots, N
$$

The sinusoidal amplitude, frequency and phase are denoted by $A, \omega$ and $\phi$, respectively, and they are considered as deterministic but unknown constants. While the noise $q_{n}$ is assumed to be a zero-mean complex white process of the form $q_{n}=u_{n}+j v_{n}$, where $u_{n}$ and $v_{n}$ are zero-mean real white processes with identical but unknown variances of $\sigma^{2} / 2$ and uncorrelated with each other. Although estimating the single frequency is a fundamental and well-studied problem, efforts have continually been made [1]-[9] to derive estimators that can attain high estimation performance but with low computational cost. Our objective is also to estimate $\omega \in(-\pi, \pi)$ accurately in a computationally simple manner, from the $N$ discrete-time noisy measurements of $\left\{x_{n}\right\}$.

In the presence of white Gaussian noise, the maximum likelihood (ML) estimate of frequency is obtained from the periodogram maximum [10] but it involves extensive computations. To avoid high computational requirement, autocorrelation or linear prediction (LP) [1], and phased-based [2] approaches are widely used choices. Although they are similar in the sense that they both extract angle information, their basic distinction is that the former utilizes the phase of the autocorrelation function of $\left\{x_{n}\right\}$, denoted by $R_{x x}(l)$, where $l$ is the lag, while the latter considers the signal phase to achieve frequency estimation. Founded on [1]-[2], many computationally efficient frequency estimators with suboptimal performance have been proposed in the literature, to name but a few, [3]-[9]. Kay [3] has proposed the so-called weighted linear predictor (WLP) frequency estimator, which introduces different weights in computing a generalized version of $R_{x x}(1)$. Frequency estimation from a set of $\left\{R_{x x}(l)\right\}$ has been investigated in [4], [6], [8]. Recently, Brown and Wang [9] have suggested to use LP together with low-pass filtering, decimation and heterodyning iteratively for single frequency estimation. On the other hand, an alternative to [2] is devised by using the differenced phase data [3], which is known as weighted phase averager (WPA), and this technique has been extended via the use of simple low-pass filtering and a set of filter banks in [5] and [7], respectively. However, as discussed in [9], most of these computationally attractive schemes have the demerits of poor threshold performance, non-uniform estimation performance across the admissible frequency range, limited frequency operation range and/or requirement of phase unwrapping which becomes prone to errors at low signal-to-noise ratio (SNR) conditions.

In this work, we aim to develop a computationally simple and accurate frequency estimation approach which does not have the above drawbacks. The rest of the paper is organized as follows. Based on the LP property of a complex tone and weighted least squares (WLS), we derive a generalized version of the WLP frequency estimator in Section 2. Mean and variance analysis of the frequency estimate is also provided. In Section 3, we extend the proposed technique to two-dimensional (2-D) single-tone frequency estimation. Numerical examples are presented in Section 4 to corroborate the analytical calculation and to evaluate algorithm performance by comparing with WLP and WPA as well as Cramér-Rao lower bound (CRLB). Finally, conclusions are drawn in Section 5. 


\section{GENERALIZED WEIGHTED LINEAR PREDICTOR}

The LP property of $s_{n}$ can be expressed as

$$
s_{n}=\rho s_{n-1}, \quad \rho=e^{j \omega}
$$

Based on (2), the LP error is

$$
\epsilon_{n}=x_{n}-\tilde{\rho} x_{n-1}, \quad n=2,3, \cdots, N
$$

where $\tilde{\rho}$ is a variable corresponds to $\rho$. Expressing (3) into vector form yields

$$
\boldsymbol{\epsilon}=\mathbf{X}_{1}-\tilde{\rho} \mathbf{X}_{\mathbf{2}}
$$

where

$$
\begin{array}{r}
\boldsymbol{\epsilon}=\left[\epsilon_{N}, \epsilon_{N-1}, \cdots, \epsilon_{2}\right]^{T} \\
\mathbf{X}_{\mathbf{1}}=\left[x_{N}, x_{N-1}, \cdots, x_{2}\right]^{T} \\
\mathbf{X}_{\mathbf{2}}=\left[x_{N-1}, x_{N-2}, \cdots, x_{1}\right]^{T}
\end{array}
$$

with $T$ denotes the transpose operation. The WLS cost function constructed from the LP error is then

$$
J(\tilde{\rho})=\boldsymbol{\epsilon}^{H} \mathbf{W} \boldsymbol{\epsilon}=\left(\mathbf{X}_{\mathbf{1}}-\tilde{\rho} \mathbf{X}_{\mathbf{2}}\right)^{H} \mathbf{W}\left(\mathbf{X}_{\mathbf{1}}-\tilde{\rho} \mathbf{X}_{\mathbf{2}}\right)
$$

where $H$ represents the conjugate transpose and $\mathbf{W} \in$ $\mathcal{C}^{(N-1) \times(N-1)}$ is a weighting matrix which satisfies $\mathbf{W}=$ $\mathbf{W}^{H}$. Differentiating $J(\tilde{\rho})$ with respect to $\tilde{\rho}$ and then setting the resultant expression to zero, we get the WLS estimate of $\omega$, denoted by $\hat{\omega}$ :

$$
\hat{\omega}=L\left(\frac{\mathbf{X}_{\mathbf{2}}{ }^{H} \mathbf{W} \mathbf{X}_{\mathbf{1}}}{\mathbf{X}_{\mathbf{2}}{ }^{H} \mathbf{W} \mathbf{X}_{\mathbf{2}}}\right)
$$

where $\angle($.$) represents the phase angle in [-\pi, \pi)$. An ideal choice of $\mathbf{W}$ is given by the Markov estimate:

$$
\mathbf{W}_{\mathbf{o}}=\sigma^{2}\left[E\left\{\boldsymbol{\epsilon}_{q} \boldsymbol{\epsilon}_{q}^{H}\right\}\right]^{-1}
$$

where $\mathbf{W}_{\mathbf{o}}{ }^{-1}$ is of the form:

$$
\left[\begin{array}{cccccc}
2 & -e^{j \omega} & 0 & 0 & \cdots & 0 \\
-e^{-j \omega} & 2 & -e^{j \omega} & 0 & \cdots & 0 \\
\vdots & \vdots & \vdots & \vdots & \vdots & \vdots \\
0 & \cdots & 0 & -e^{-j \omega} & 2 & -e^{j \omega} \\
0 & 0 & \cdots & 0 & -e^{-j \omega} & 2
\end{array}\right]
$$

with $\boldsymbol{\epsilon}_{q}=\left[q_{N}-e^{j \omega} q_{N-1}, q_{N-1}-e^{j \omega} q_{N-2}, \cdots, q_{2}-\right.$ $\left.e^{j \omega} q_{1}\right]^{T}, E$ is the expectation operator and ${ }^{-1}$ denotes matrix inverse. It is noteworthy that $e^{j \omega}$ is used instead of $\rho$ in $\boldsymbol{\epsilon}_{q}$. In doing so, we are able to obtain the closed-form expression of $\mathbf{W}_{\mathbf{o}}$ and its $(m, n)$ entry is shown to be

$$
\left[\mathbf{W}_{\mathbf{o}}\right]_{m, n}=\frac{N \min (m, n)-m n}{N} e^{j(n-m) \omega}
$$

where $\min (m, n)=m$ if $m<n$ and it is equal to $n$ otherwise, for $1 \leq m \leq N-1$ and $1 \leq n \leq N-1$. It should be noted that the factor $1 / N$ in (8) can be omitted due to the cancelation appeared in the numerator and the denominator in (6). Furthermore, we notice that $\mathbf{W}_{\mathbf{o}}$ is a positive-definite Hermitian matrix, which implies that $\mathbf{X}_{\mathbf{2}}{ }^{H} \mathbf{W}_{\mathbf{o}} \mathbf{X}_{\mathbf{2}}$ is positive and thus of zero phase. As a result, the frequency estimate shown in (6) can be simplified as:

$$
\hat{\omega}=L\left(\mathbf{X}_{\mathbf{2}}{ }^{H} \mathbf{W}_{\mathbf{o}} \mathbf{X}_{\mathbf{1}}\right)
$$

As the ideal $\mathbf{W}$ is a function of the unknown frequency parameter, we propose to use a relaxation algorithm for iterative frequency estimation and the procedure is summarized as follows:

(i) Compute an initial value of $\hat{\omega}$ using the WLP frequency estimate.

(ii) Use $\hat{\omega}$ to construct $\mathbf{W}_{\mathbf{o}}$ in (8).

(iii) Calculate an updated $\hat{\omega}$ using (9).

(iv) Repeat Steps (ii)-(iii) until parameter convergence.

Expanding (9) in a scalar form via summing up the diagonals of $\mathbf{W}_{\mathbf{o}}$ one by one yields

$$
\begin{aligned}
\hat{\omega}= & L\left(\sum_{m=1}^{N-1} \sum_{n=1}^{N-1} x_{N-m}^{*}\left[\mathbf{W}_{\mathbf{o}}\right]_{m, n} x_{N+1-n}\right) \\
= & L\left(\sum_{m=1}^{N-1} x_{m}^{*}\left[\mathbf{W}_{\mathbf{o}}\right]_{m, m} x_{m+1}+\right. \\
& \sum_{l=1}^{N-2} \sum_{k=l+1}^{N-1} x_{N-k}^{*}\left[\mathbf{W}_{\mathbf{o}}\right]_{k, k-l} x_{N-k+l+1}+ \\
& \left.\sum_{l=2-N}^{-1} \sum_{k=1}^{N+l-1} x_{N-k}^{*}\left[\mathbf{W}_{\mathbf{o}}\right]_{k, k-l} x_{N-k+l+1}\right)
\end{aligned}
$$

where $*$ represents complex conjugate and

$$
\left[\mathbf{W}_{\mathbf{o}}\right]_{m, m}=\frac{N}{4}\left(1-\left[\frac{m-1-\left(\frac{N}{2}-1\right)}{\frac{N}{2}}\right]^{2}\right)
$$

By comparing [3] and (11), it can be easily shown that the first component of (10), that is, the sum which utilizes the first-order autocorrelations, is identical to the WLP approach with the same parabolic weights up to a real scalar. While the second and third components of (10) represent the higherorder as well as zero-order weighted linear prediction terms. This clearly shows how our approach, which is referred to as generalized weighted linear predictor (GWLP), generalizes the WLP. It is noteworthy to mention that (10) is also similar to autocorrelation-based methods of [4], [6], [8] which use 
different combinations of the higher-order autocorrelations. However, one major difference between the GWLP and these autocorrelation-based methods is that the latter are subject to phase unwrapping errors or have limited frequency operation range while the former is free of these demerits.

To simplify the bias and variance analysis of the GWLP frequency estimate, we assume that the ideal weighting matrix of (7) is used. Note that this assumption becomes valid when $\hat{\omega}$ approaches the true value of $\omega$, which is anticipated to occur at sufficiently large SNR and/or data length conditions. Taking the expected value of (9) with the use of the ideal weighting matrix, it can be shown that

$$
E\{\hat{\omega}\}=\omega+E\left\{L\left(e^{-j \omega} \mathbf{X}_{\mathbf{2}}{ }^{H} \mathbf{W}_{\mathbf{o}} \mathbf{X}_{\mathbf{1}}\right)\right\} \approx \omega
$$

which indicates the approximately unbiasedness of the algorithm. Expressing $\hat{\omega}=\omega+\Delta \omega$ where $\Delta \omega$ denotes the estimation error, the variance of $\hat{\omega}$, denoted by $\operatorname{var}(\hat{\omega})$, is derived as

$$
\operatorname{var}(\hat{\omega}) \approx E\left\{(\Delta \omega)^{2}\right\} \approx \frac{6}{\operatorname{SNR} N\left(N^{2}-1\right)}
$$

where $\mathrm{SNR}=A^{2} / \sigma^{2}$, which is equal to CRLB [10] for single frequency estimation in white Gaussian noise. As a result, we expect that the GWLP approach can attain optimum frequency estimation.

\section{EXTENSION TO TWO-DIMENSIONAL ESTIMATION}

The GWLP approach is extended to 2-D single-tone frequency estimation as follows. The 2-D data set can be modelled as

$$
\begin{aligned}
x_{m, n}= & \gamma e^{j(\mu m+\nu n)}+q_{m, n} \\
& m=1,2, \ldots, M, \quad n=1,2, \ldots, N
\end{aligned}
$$

where $\gamma$ is the unknown complex amplitude and $\mu \in(-\pi, \pi)$ and $\nu \in(-\pi, \pi)$ are the unknown frequencies of the 2-D tone while $q_{m, n}$ is a zero-mean complex white noise.

By considering each row of $\left\{x_{m, n}\right\}$ is a 1-D signal which is parameterized by $\nu$ only and utilizing all row information, it is straightforward to apply (10) for estimating $\nu$ :

$$
\hat{\nu}=L\left(\sum_{i=1}^{M} \sum_{m=1}^{N-1} \sum_{n=1}^{N-1} x_{i, N-m}^{*}\left[\mathbf{W}_{\mathbf{o}}\right]_{m, n} x_{i, N+1-n}\right)
$$

Alternatively, the estimate of $\mu$ is obtained in a similar manner via partitioning $\left\{x_{m, n}\right\}$ column-by-column. We just follow the iterative procedure in Section 2 to achieve 2-D frequency estimation. It is noteworthy that the same procedure can be used to extend the GWLP approach to higher dimensional frequency estimation as well.

\section{SIMULATION RESULTS}

Computer simulations had been carried out to evaluate the performance of the proposed GWLP approach in 1-D and 2$\mathrm{D}$ frequency estimation. We used 2 iterations in all GWLP algorithms because no significant improvement was observed for more iterations. The signal power was unity and we scaled the noise sequence to produce different SNRs.

Figure 1 shows the mean square frequency error (MSFE) performance versus SNR for $1-\mathrm{D}$ case at $\omega=0.1 \pi$ and $N=20$. The results of the two computationally efficient frequency estimation algorithms, namely, WLP and WPA [3] as well as the CRLB were also included. It is seen that the MSFEs of GWLP attained the CRLB for SNR $\geq 4 \mathrm{~dB}$, which agreed with our theoretical analysis. Furthermore, the WPA could approach the CRLB but its threshold SNR was higher than that of the GWLP. While it is seen that the WLP was optimum only for very high SNR conditions. Figure 2 plots the frequency versus SNR contours of MSFE for GWLP at $N=20$ in order to investigate the threshold performance in more detail. It is observed that the proposed approach had the desired property of uniform estimation performance at sufficiently high SNRs.

Figures 3 and 4 show the GWLP estimation performance for a 2-D complex single-tone versus SNR when $\mu=\nu=$ $0.1 \pi$ with $M=N=8$. The results of the $2-\mathrm{D}$ version of the WPA [11] and corresponding CRLB were also included for comparison. It is observed that the performance of the GWLP approached the CRLB for sufficiently high SNR conditions and was superior to the WPA because the former had a smaller threshold SNR.

\section{CONCLUSIONS}

A novel iterative frequency estimation approach, which is referred to as generalized weighted linear predictor (GWLP), has been developed for a complex sinusoid embedded in white noise. The proposed approach can be considered as a generalized version of Kay's weighted linear predictor frequency estimator. The GWLP derivation is based on the well known ideas of linear prediction and weighted least squares. Extension to two-dimensional frequency estimation is also studied. The optimality of the GWLP approach is demonstrated via theoretical calculation as well as computer simulations.

\section{Acknowledgement}

The work described in this paper was supported by a grant from CityU (Project No. 7001708). 


\section{References}

[1]G.W.Lank, I.S.Reed and G.E.Pollon, "A semicoherent detection and Doppler estimation statistic," IEEE Trans. Aerosp. Electron. Syst., vol.9, pp.151-165, Mar. 1973

[2] S.A.Tretter, "Estimating the frequency of a noisy sinusoid by linear regression," IEEE Trans. Inform. Theory, vol.31, pp.832-835, Nov. 1985

[3] S.Kay, "A fast and accurate single frequency estimator," IEEE Trans. Acoust., Speech, Signal Processing, vol.37, pp.1987-1990, Dec. 1989

[4] M.P.Fitz, "Further results in the fast estimation of a single frequency," IEEE Trans. Commun., vol.42, pp.862-864, Feb. 1994

[5] D.Kim, M.Narasimha and D.Cox, "An improved single frequency estimator," IEEE Signal Processing Lett., vol.3, pp.212-214, July 1996

[6] P.Händel, "Markov-based single-tone frequency estimation," IEEE Trans. Circuits Syst. II, vol.45, pp.230-232, Feb. 1998

[7] M.L.Fowler and J.A.Johnson, "Extending the threshold and frequency range for phase-based frequency estimation," IEEE Trans. Signal Processing, pp.2857-2863, Oct. 1999

[8] B.Völcker and P.Händel, "Frequency estimation from proper sets of correlations," IEEE Trans. Signal Processing, vol.50, pp.791-802, Apr. 2002

[9] T.Brown and M.M.Wang, "An iterative algorithm for singlefrequency estimation," IEEE Trans. Signal Processing, vol.50, pp.2671-2682, Nov. 2002

[10] D.C.Rife and R.R.Boorstyn, "Single tone parameter estimation from discrete-time observations," IEEE Trans. Inform. Theory, vol.20, pp.591-598, Sept. 1974

[11] Kay and R.Nekovei, "An efficient two-dimensional frequency estimator," IEEE Trans. Acoust., Speech, Signal Processing, vol.38, pp.1807-1809, Oct. 1990

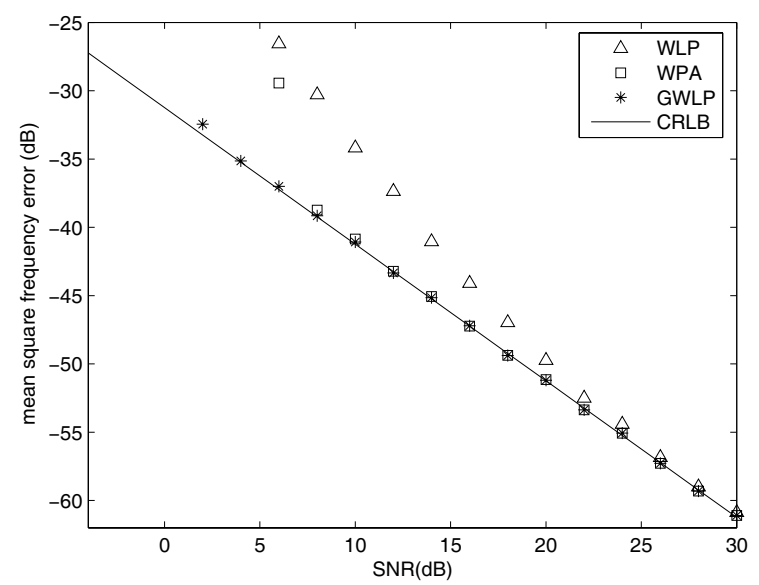

Fig. 1. Mean square error of $\omega$ versus SNR

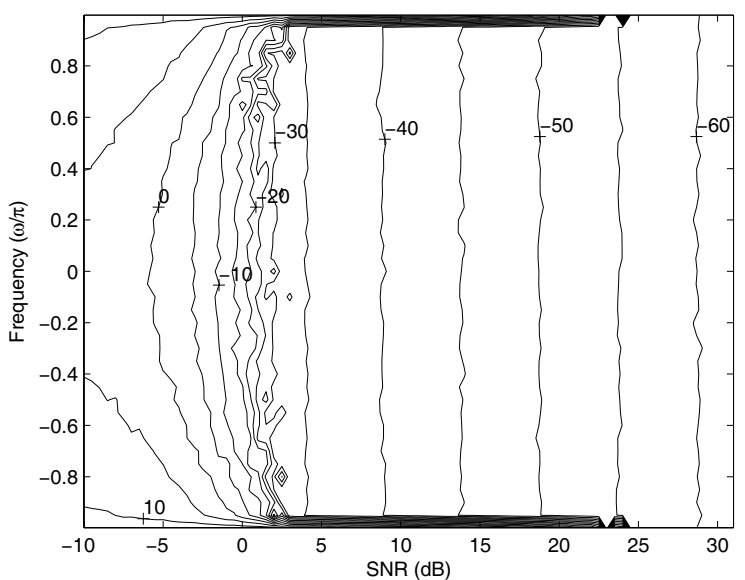

Fig. 2. Contour plot of GWLP

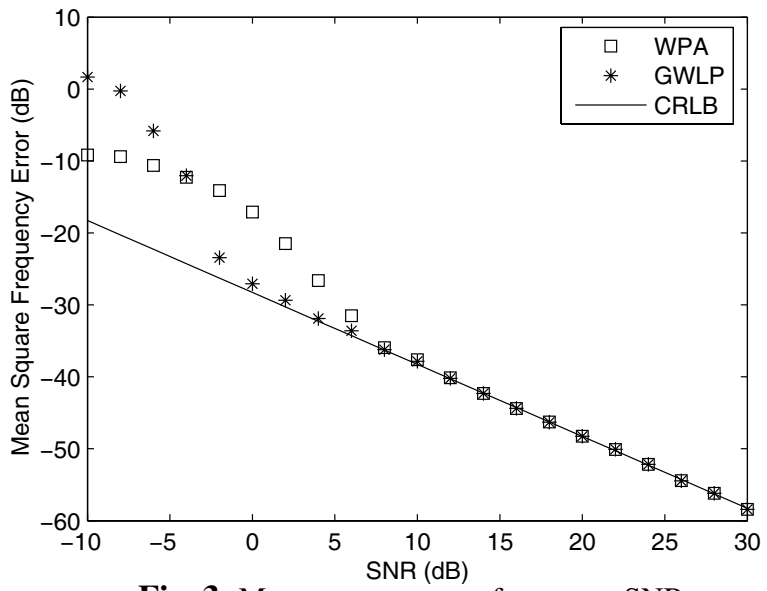

Fig. 3. Mean square error of $\mu$ versus SNR

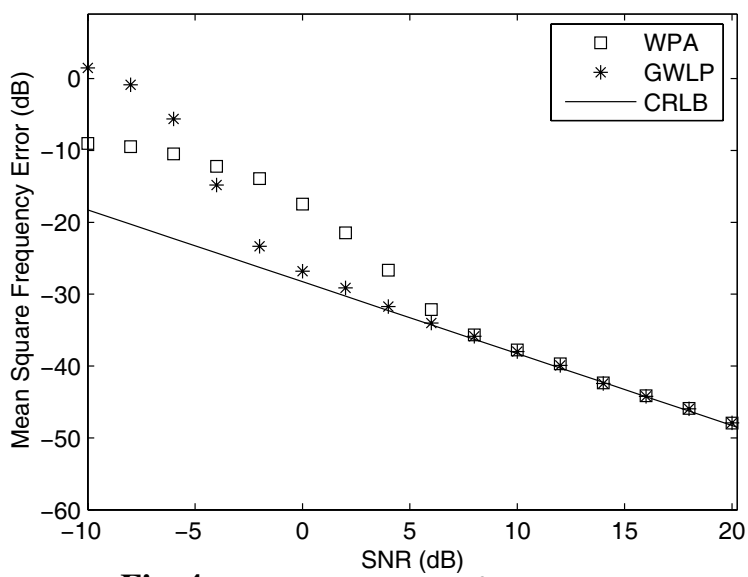

Fig. 4. Mean square error of $\nu$ versus SNR 